\title{
Calcul de résolvantes avec les modules de Cauchy
}

\author{
Nicolas Rennert et Annick Valibouze
}

\section{TABLE DES MATIÈRES}

1. Introduction

2. Notations et définitions

3. Polynômes caractéristiques et résolvantes

4. Calcul du polynôme caractéristique dans l'algèbre de décomposition universelle

5. Réductions avec des bases de Gröbner

6. Calcul de la résolvante absolue

7. Généralisation aux multi-résolvantes

8. Implantation

9. Temps de calculs et comparaisons avec SYM

10. Conclusions

Disponibilité electronique

Bibliographie

Les auteurs remercient le projet GALOIS du GDR de Calcul Formel MEDICIS.
Cet article décrit un algorithme nouveau et efficace pour calculer des polynômes caractéristiques d'endomorphismes dans un algèbre quotient en utilisant les modules de Cauchy. Cet algorithme est ensuite utilisé pour calculer des résolvantes et des multi-résolvantes absolues, outils de base de la théorie des corps et donc aussi de la théorie de Galois constructive.

We give a new and efficient algorithm to compute some characteristic polynomials using Cauchy modules. This algorithm is used for the computation of absolute resolvents and multiresolvents which are essential tools in constructive Galois theory.

\section{INTRODUCTION}

La résolvante est l'outil de base de la théorie de Galois effective dont le calcul à la main s'avère vite impossible. Ainsi, chercher à diminuer le temps consacré à son calcul améliore celui du calcul du groupe de Galois d'un polynôme. Historiquement les premiers à réaliser de tels calculs à l'aide de l'outil informatique, furent R. P. Stauduhar [1973] et K. Girstmair [1983] utilisant pour le premier une méthode basée sur des calculs numériques. Bien que le résultat ne soit pas certifié, cette méthode s'avère être la plus rapide dans le cas général. Le logiciel GALP [Eichenlaub et Olivier 1996] basée sur la librairie PARI/GP, utilise ces techniques pour déterminer le groupe de Galois de polynômes.

Les méthodes algébriques ont en revanche l'avantage d'être exactes. Parmi elles, les méthodes basées sur les fonctions symétriques s'avèrent efficaces pour des invariants particuliers; voir [Valibouze 1989]. Cet article s'intéresse aux algorithmes qui calculent des résolvantes pour un invariant quelconque et qui sont basés sur l'opération de résultant.

Le résultant est un outil particulièrement puissant pour manipuler algébriquement les racines de polynômes qui depuis Lagrange a servi pour le calcul de la résolvante; voir [Lagrange 1770]. Le premier qui 
sur machine a utilisé le résultant pour le calcul de résolvantes (absolues) est L. H. Soicher [1984] pour des invariants linéaires.

Jusqu'à présent les méthodes basées sur le résultant pour calculer la résolvante (absolue) se heurtaient à deux défauts: apparition de facteurs et de puissances "parasites" qui augmentent considérablement la taille des polynômes nécessaires et réduisent donc l'efficacité. Cet article présente un algorithme simple et rapide (voir théorème 4.7) qui calcule, sans formation de facteurs parasites, le polynôme caractéristique d'un endomorphisme multiplicatif associé à un invariant quelconque donné. Le polynôme caractéristique est une puissance de la résolvante pour ce même invariant. Ainsi le problème des facteurs "parasites" est résolu, reste encore celui de la puissance "parasite". L'algorithme proposé utilise essentiellement le résultant et les modules de Cauchy qui forment une base de Gröbner réduite de l'idéal des relations symétriques (pour l'ordre lexicographique). Si les calculs du théorème 4.7 sont réalisés modulo l'idéal des relations symétriques alors la puissances à laquelle nous obtenons la résolvante est réduite et les calculs intermédiaires accélérés. Si les calculs modulaires n'aboutissent pas à la résolvantes mais à une puissance strictement positive (ce qui est prévisible a priori alors nous utilisons alors le résultat de F. Lehobey [1997] pour éliminer ces puissances en cours de calcul (ce travail fait référence aux résultats de article). Ainsi le problème de la puissance parasite est également résolu (voir algo $6.10)$.

Dans le paragraphe 7 l'algorithme de calcul des résolvantes est naturellement généralisé au cas des multi-résolvantes - c'est-à-dire les résolvantes relatives à un produit de groupes symétriques. Les derniers paragraphes sont consacrés à l'implantation de cette méthode, la comparaison avec d'autres algorithmes algébriques ainsi que des améliorations.

\section{NOTATIONS ET DÉFINITIONS}

- $\mathcal{K}$ désigne un corps de caractéristique 0 ;

- $x$ est une variable indéterminée sur $\mathcal{K}$;

- $f$ est un polynôme unitaire de $\mathcal{K}[x]$ de degré $n$;

$-x_{1}, \ldots, x_{n}$ sont des variables indéterminées sur $\mathcal{K}$;

- $\mathcal{K}\left[x_{1}, \ldots, x_{n}\right]$ désigne l'anneau des polynômes en les variables $x_{1}, \ldots, x_{n}$ et à coefficients dans $\mathcal{K}$;
- $\mathcal{K}\left(x_{1}, \ldots, x_{n}\right)$ désigne le corps des fractions de $\mathcal{K}\left[x_{1}, \ldots, x_{n}\right]$;

- $\Psi$ est un polynôme appartenant à $\mathcal{K}\left[x_{1}, \ldots, x_{n}\right]$;

- $\alpha=\left(\alpha_{1}, \ldots, \alpha_{n}\right)$ est une liste (ordonnée) formée des $n$ racines du polynôme $f$ dans une clôture algébrique $\hat{\mathcal{K}}$ de $\mathcal{K}$;

- $\Psi(\alpha)=\Psi\left(\alpha_{1}, \ldots, \alpha_{n}\right)$;

- $\mathfrak{S}_{n}$ désigne le groupe symétrique de degré $n$.

Definition 2.1. Le polynôme $\Psi$ est dit d'arité $m$ si $m$ est le plus petit entier $j$ tel qu'il existe $j$ entiers distincts $i_{1}, \ldots, i_{j}$ vérifiant $\Psi \in \mathcal{K}\left[x_{i_{1}}, \ldots, x_{i_{j}}\right]$.

Definition 2.2. Si $\Psi(\alpha)=0$ alors $\Psi$ est appelée une $\alpha$-relation.

Definition 2.3. L'action du groupe symétrique $\mathfrak{S}_{n}$ sur $\Psi$ est définie par:

$$
\tau . \Psi=\Psi\left(x_{\tau(1)}, \ldots, x_{\tau(n)}\right) \text { pour tout } \tau \in \mathfrak{S}_{n} .
$$

Definition 2.4. L'action du groupe symétrique $\mathfrak{S}_{n}$ sur $\hat{\mathcal{K}}^{n}$ est définie, pour tout $\sigma \in \mathfrak{S}_{n}$ et tout

$$
\beta=\left(\beta_{1}, \ldots, \beta_{n}\right) \in \hat{\mathcal{K}}^{n},
$$

$\operatorname{par} \beta_{\sigma}=\left(\beta_{\sigma(1)}, \beta_{\sigma(2)}, \ldots, \beta_{\sigma(n)}\right)$.

Definition 2.5. L'orbite de $\Psi$ sous l'action d'un sousgroupe $L$ de $\mathfrak{S}_{n}$, notée $L . \Psi$, est définie par:

$$
L . \Psi=\{\tau . \Psi \mid \tau \in L\}
$$

Definition 2.6. Soient $L$ et $H$ deux sous-groupes de $\mathfrak{S}_{n}$ tels que $L$ contienne $H$. Le polynôme $\Psi$ est dit $L$-primitif $H$-invariant si $H$ est le stabilisateur de $\Psi$ dans $L$ :

$$
H=\operatorname{Stab}_{L}(\Psi)=\{\tau \in L \mid \tau . \Psi=\Psi\} .
$$

Lorsque $L=\mathfrak{S}_{n}$, l'invariant $\Psi$ est dit également $H$-invariant primitif.

Exemple 2.7. Soit $H=\mathfrak{S}_{2} \times \mathfrak{S}_{n-2}$. Le polynôme $\Psi=$ $x_{1}+x_{2}$ est un $\mathfrak{S}_{n}$-primitif $H$-invariant.

Definition 2.8. Soient deux sous-groupes $L$ et $H$ du groupe symétrique $\mathfrak{S}_{n}$ tels que $L$ contienne $H$. On appelera l'ensemble $\left\{\tau_{1}, \ldots, \tau_{e}\right\}$ une transversale $\grave{a}$ gauche de $L \bmod H$ si $\tau_{1} H, \ldots, \tau_{e} H$ sont les différentes classes à gauches de $L \bmod H$.

Definition 2.9. Pour $i=0, \ldots, n$, la $i$-ième fonction symétrique élémentaire en $x_{1}, \ldots, x_{n}$, notée $e_{i}$, est définie $\operatorname{par} e_{0}=1$ et

$$
e_{i}=\sum_{m \in \mathfrak{S}_{n} \cdot\left(x_{1} \cdots x_{i}\right)} m \quad \text { pour } i \geq 1 .
$$


Remarque 2.10. Rappelons que

$f(x)=x^{n}-e_{1}(\alpha) x^{n-1}+e_{2}(\alpha) x^{n-2}+\cdots+(-1)^{n} e_{n}(\alpha)$.

Definition 2.11. Les $n$ fonctions interpolaires introduites par Ampère [1826] vérifient:

$$
f_{i} \in \mathcal{K}\left[x_{i+1}, \ldots, x_{n}\right][x] \quad \text { et } \quad \operatorname{deg}_{x}\left(f_{i}\right)=i
$$

et sont définies par $f_{n}(x)=f(x)$ et

$$
f_{i}(x)=f_{i}\left(x, x_{i+1}, \ldots, x_{n}\right)=\frac{f_{i+1}(x)-f_{i+1}\left(x_{i+1}\right)}{x-x_{i+1}}
$$

pour $n-1 \geq i \geq 1$.

Definition 2.12. Les polynômes $f_{1}\left(x_{1}\right), f_{2}\left(x_{2}\right), \ldots$, $f_{n}\left(x_{n}\right)$ sont appelés les modules de Cauchy associés au polynôme $f$.

Definition 2.13. Soit $L$ un sous-groupe du groupe symétrique $\mathfrak{S}_{n}$. L'idéal

$$
I_{\alpha}^{L}=\left\{r \in \mathcal{K}\left[x_{1}, \ldots, x_{n}\right] \mid(\forall \sigma \in L)(\sigma . r)(\alpha)=0\right\}
$$

est un idéal radical appelé idéal des $\alpha$-relations invariantes par $L$.

Definition 2.14. L'idéal $I_{\alpha}^{\mathfrak{S}_{n}}$, noté $\mathcal{J}$, est connu sous le nom d'idéal des relations symétriques entre les racines du polynôme $f$.

Definition 2.15. Soit $I_{n}$ le groupe identité dans $\mathfrak{S}_{n}$. L'idéal $I_{\alpha}^{I_{n}}$, noté $I_{\alpha}$, est connu sous le nom d'idéal des $\alpha$-relations.

Remarque 2.16. Le quotient $\mathcal{K}\left[x_{1}, \ldots, x_{n}\right] / \mathcal{J}$ est appelé algèbre de décomposition universelle.

Definition 2.17. Le sous-groupe $G_{\alpha}$ du groupe symétrique $\mathfrak{S}_{n}$ défini par

$$
G_{\alpha}=\left\{\sigma \in \mathfrak{S}_{n} \mid\left(\forall r \in \mathcal{J}_{\alpha}\right)(\sigma . r)(\alpha)=0\right\}
$$

est connu abusivement sous le nom de groupe de Galois de $f$. On le nommera groupe de Galois de $\alpha$.

Notation 2.18. Soit $I$ un idéal de $\mathcal{K}\left[x_{1}, \ldots, x_{n}\right]$. Pour $\Theta \in \mathcal{K}\left[x_{1}, \ldots, x_{n}\right]$ notons $\hat{\Theta}$ la multiplication par la classe de $\Theta$ dans l'anneau quotient $\mathcal{K}\left[x_{1}, \ldots, x_{n}\right] / I$. C'est un endomorphisme du $\mathcal{K}$-espace vectoriel

$$
\mathcal{K}\left[x_{1}, \ldots, x_{n}\right] / I .
$$

Le polynôme caractéristique de l'endomorphisme $\hat{\Theta}$ sera noté $\chi_{\Theta, I}$. Si $I=\mathcal{J}$ alors il sera noté simplement $\chi_{\Theta}$.
Definition 2.19. Soit $L$ un sous-groupe du groupe symétrique $\mathfrak{S}_{n}$ tel que $L$ contienne le groupe de Galois $G_{\alpha}$ du polynôme $f$. La $L$-relative résolvante de $f$ par $\Psi$ (associée à la numérotation $\alpha$ ), notée $\mathcal{L}_{\Psi, L, \alpha}$, est le polynôme de $\mathcal{K}[T]$ défini comme suit:

$$
\mathcal{L}_{\Psi, L, \alpha}(T)=\prod_{\Theta \in L . \Psi}(T-\Theta(\alpha)) .
$$

Si $L=\mathfrak{S}_{n}$, la résolvante est notée $\mathcal{L}_{\Psi, f}$ et appelée résolvante (absolue) de $f$ par $\Psi$.

Comme le groupe $L$ contient le groupe de Galois $G_{\alpha}$ et que le corps $\mathcal{K}$ est parfait, par la théorie de Galois, la résolvante appartient bien au corps $\mathcal{K}[T]$.

\section{POLYNÔMES CARACTÉRISTIQUES ET RÉSOLVANTES}

Dans ce paragraphe, le polynôme $f$ est supposé séparable (ses racines sont distinctes deux à deux) et $L$ désigne un sous-groupe de $\mathfrak{S}_{n}$ qui contient le groupe de Galois $G_{\alpha}$.

Proposition 3.1. La variété de l'idéal $I_{\alpha}^{L}$ dans une clôture algébriques de $\mathcal{K}$, notée $V\left(I_{\alpha}^{L}\right)$, est l'orbite de la liste $\alpha$ sous l'action du groupe $L$ :

$$
V\left(I_{\alpha}^{L}\right)=\left\{\alpha_{\sigma} \mid \sigma \in L\right\} .
$$

Comme le polynôme $f$ est séparable, le cardinal de cette variété est celui du groupe $L$.

Démonstration. La preuve est simple. Voir [Valibouze 1999].

Pour un idéal $I$ radical, le théorème de Stickelberger donne cette expression explicite du polynôme caractéristique de l'endomorphisme $\hat{\Psi}$ associé à un idéal $I$ (voir notation 2.18) :

$$
\chi_{\Psi, I}(T)=\prod_{\beta \in V(I)}(T-\Psi(\beta)) .
$$

Comme l'idéal $I_{\alpha}^{L}$ est radical et que le polynôme $f$ est séparable, il vient:

$$
\chi_{\Psi, I_{\alpha}^{L}}(T)=\prod_{\sigma \in L}(T-(\sigma . \Psi)(\alpha)) .
$$

En particulier pour $I=\mathcal{J}$, l'idéal des relations symétriques, on a :

$$
\chi_{\Psi}(T)=\prod_{\sigma \in \mathfrak{S}_{n}}(T-(\sigma . \Psi)(\alpha)) .
$$


Soient $H$ un sous-groupe du groupe $L$ et $\mathcal{T}$ une transversale à gauche de $L \bmod H$. Si $\Psi$ est un $L$ primitif $H$-invariant nous avons l'identité suivante bien connue :

$$
\mathcal{L}_{\Psi, L, \alpha}(T)=\prod_{\tau \in \mathcal{T}}(T-(\tau . \Psi)(\alpha)) .
$$

En effet, pour tout $\tau \in \mathcal{T}$ et pour tout $h \in H$, on a $\tau . \Psi=\tau h . \Psi$. De plus si $\sigma \in \mathcal{T}$ avec $\sigma \neq \tau$, alors $\sigma . \Psi \neq \tau . \Psi$.

Et nous obtenons (voir [Arnaudiès et Valibouze 1997] pour $\left.L=\mathfrak{S}_{n}\right)$ :

$$
\chi_{\Psi, I_{\alpha}^{L}}=\left(\mathcal{L}_{\Psi, L, \alpha}\right)^{\operatorname{card}(H)}
$$

où il apparaît que le polynôme caractéristique s'exprime comme une puissance de la résolvante absolue.

Dans le prochain paragraphe nous verrons comment se calcule le polynôme caractéristique $\chi_{\Psi}$ lié à l'algèbre de décomposition universelle, puis dans les suivants nous chercherons à calculer la résolvante absolue à partir de la trame de l'algorithme de calcul du polynôme caractéristique.

\section{CALCUL DU POLYNÔME CARACTÉRISTIQUE DANS L'ALGÈBRE DE DÉCOMPOSITION UNIVERSELLE}

Ce paragraphe énonce la formule de J. M. Arnaudiès et l'algorithme de J. L. Lagrange puis il termine par un nouvel algorithme inspiré de celui de Lagrange.

Rappel 4.1. Soient $u$ et $v$ deux polynômes de $\mathcal{K}[x]$, avec $u(x)=a \prod_{i=1}^{m}\left(x-\beta_{i}\right)$ où $\beta_{1}, \ldots, \beta_{m}$ appartiennent à une clôture algébrique de $\mathcal{K}$. Le résultant en $x$ des deux polynômes $u$ et $v$, noté $\operatorname{Res}_{x}(u, v)$, peut s'exprimer ainsi :

$$
\operatorname{Res}_{x}(u, v)=a^{\operatorname{deg}(v)} \prod_{i=1}^{m} v\left(\beta_{i}\right) .
$$

\section{A. La méthode d'Arnaudiès}

la méthode proposée dans [Arnaudiès 1992] est basée sur le résultant à $n$ variables.

Notons $x_{n+1}$ une indéterminée supplémentaire et $d$ le degré total de $\Psi$ en $x_{1}, \ldots, x_{n}$. L'homogénéisé de $\Psi$, noté $\Psi^{*}$, est donné par:

$$
\Psi^{*}=x_{n+1}^{d} \Psi\left(\frac{x_{1}}{x_{n+1}}, \ldots, \frac{x_{n}}{x_{n+1}}\right) .
$$

Notons $\operatorname{Res}\left(g_{1}, \ldots, g_{n}\right)$ le résultant de $n$ polynômes $g_{1}, \ldots, g_{n}$ homogènes non constants à $n$ variables.

Théorème 4.2 (Arnaudiès). Posons $s_{i}=e_{i}-e_{i}(\alpha)$ pour $i=1, \ldots, n$. Alors le polynôme caractéristique est donné par:

$$
\chi_{\Psi}(T)=\operatorname{Res}\left(s_{1}^{*}, \ldots, s_{n}^{*}, T x_{n+1}^{d}-\Psi^{*}\right) .
$$

Ce théorème démontré dans [Arnaudiès 1992] se déduit de la formule de Poisson-Perron: voir [Arnaudiès 1989, p. 243]. Le calcul de résultant à $n$ variables s'avère très coûteux, cette méthode n'est donc pas utilisable en pratique.

\section{B. La méthode de Lagrange}

Ce paragraphe traduit en terme de résultants, la méthode que J. L. Lagrange [1770] proposait pour calculer la résolvante absolue $\mathcal{L}_{\Psi, f}$ et qui calcule le polynôme caractéristique.

Soit $\left(U_{i}\right)_{0 \leq i \leq n}$ la suite finie définie inductivement par $U_{0}\left(T, x_{1}, \ldots, x_{n}\right)=T-\Psi\left(x_{1}, \ldots, x_{n}\right)$ et

$$
\begin{aligned}
& U_{i}\left(T, x_{i+1}, \ldots, x_{n}\right)= \\
& \operatorname{Res}_{x_{i}}\left(f\left(x_{i}, \ldots, x_{n}\right), U_{i-1}\left(T, x_{i}, \ldots, x_{n}\right)\right)
\end{aligned}
$$

pour $1 \leq i \leq n$. Alors, d'après le rappel 4.1 et puisque $f$ est unitaire:

$$
U_{n}(T)=\prod_{i_{n}=1}^{n} \prod_{i_{n}-1=1}^{n} \cdots \prod_{i_{1}=1}^{n}\left(T-\Psi\left(\alpha_{i_{1}}, \ldots, \alpha_{i_{n}}\right)\right) .
$$

Les facteurs sur $\mathcal{K}$ du polynôme $U_{n}(T)$ sont

- le polynôme caractéristique $\chi_{\Psi}$;

- des facteurs dits parasites provenant des égalités deux à deux, trois à trois, $\ldots, n$ à $n$ des indices apparaissant dans les produits. Effectivement les nombres algébriques tels $\Psi\left(\alpha_{1}, \alpha_{1}, \alpha_{3}, \ldots, \alpha_{n}\right)$, $\Psi\left(\alpha_{1}, \alpha_{1}, \alpha_{1}, \alpha_{3}, \ldots, \alpha_{n}\right), \ldots, \Psi\left(\alpha_{1}, \alpha_{1}, \ldots, \alpha_{1}\right)$ sont racines de $U_{n}(T)$ et ne sont pas racines du polynôme caractéristique.

Ces facteurs parasites étaient déjà signalés par J. L. Lagrange. Ce sont des polynômes caractéristiques associés à des invariants d'arité strictement inférieure à celle de l'invariant $\Psi$. Par décroissance stricte de l'arité, il est donc toujours possible de déduire le polynôme caractéristique $\chi_{\Psi}$ à partir du polynôme $U_{n}$. Donnons un exemple explicite: 
Exemple 4.3. Choisissons l'invariant $\Psi=x_{1}+2 x_{2}$ et $n=2$. Alors

$$
U_{2}(T)=\chi_{\Psi} \cdot \chi_{3 x_{1}}
$$

où $\chi_{3 x_{1}}=\operatorname{Res}_{x}(f(x), T-3 x)$. De plus $\mathcal{L}_{\Psi, f}=\chi_{\Psi}$ puisque $\Psi$ est un $I_{2}$-invariant primitif.

La méthode proposée au paragraphe $4 \mathrm{C}$ évite la formation des facteurs parasites et calcule donc directement le polynôme caractéristique. Lagrange, qui cherchait à calculer la résolvante, signalait également la puissance parasite provenant des symétries de l'invariant $\Psi$ et que nous retrouvons dans la formule (3-2). Il est possible de diminuer la puissance en cours de calcul (voir le paragraphe 6 consacré au calcul de la résolvante).

\section{C. Méthode des modules de Cauchy}

Notons $f_{1}, \ldots, f_{n}$ les modules de Cauchy associés au polynôme $f$ (voir définition 2.12).

Definition 4.4. Soient $r$ un entier positif et $s$ un entier compris entre 1 et $n$. La $r$-ième fonction symétrique complète, notée $h_{r}\left(x_{1}, \ldots, x_{s}\right)$, est la somme des monômes de degré total $r$ en $x_{1}, \ldots, x_{s}$, avec $h_{0}\left(x_{1}, \ldots, x_{s}\right)=1$.

Le théorème suivant permet de les calculer efficacement il se démontre facilement.

Théorème 4.5 (Machì-Valibouze). Soit le polynôme

$$
f(x)=x^{n}+a_{1} x^{n-1}+a_{2} x^{n-2}+\cdots+a_{n}
$$

$\grave{a}$ coefficients dans $\mathcal{K}$. Posons $a_{0}=1$. Alors, pour $1 \leq r \leq n$, le r-ième module de Cauchy associé à $f$ est donné par:

$$
f_{r}\left(x_{r}\right)=\sum_{i=0}^{r} h_{i}\left(x_{r}, \ldots, x_{n}\right) a_{r-i} .
$$

En particulier $f_{n}\left(x_{n}\right)=\sum_{i=0}^{n} h_{i}\left(x_{n}\right) a_{n-i}=f\left(x_{n}\right)$ et $f_{1}\left(x_{1}\right)=h_{1}\left(x_{1}, \ldots, x_{n}\right)+a_{1}$.

Remarque 4.6. A. Cauchy donna la formule jusqu'à $n=4$. Une démonstration simple proposée par A. Lascoux peut-être faite à l'aide des différences divisées; voir [Lascoux et Pragacz 1988].

Le théorème suivant donne une méthode explicite du calcul du polynôme caractéristique:
Théorème 4.7. Soit la suite finie $R_{0}, R_{1}, \ldots, R_{n}$ définie inductivement par $R_{0}\left(T, x_{1}, \ldots, x_{n}\right)=T-\Psi$ et

$R_{i}\left(T, x_{i+1}, \ldots, x_{n}\right)=$

$$
\operatorname{Res}_{x_{i}}\left(f_{i}\left(x_{i}\right), R_{i-1}\left(T, x_{i}, \ldots, x_{n}\right)\right)
$$

pour $1 \leq i \leq n$. Alors le polynôme caractéristique est donné par

$$
\chi_{\Psi}(T)=R_{n}(T) .
$$

Démonstration. Nous montrons le théorème par récurrence sur $n$, le degré du polynôme $f$.

Pour $n=1, f=\left(x-\alpha_{1}\right)$, comme le polynôme $f$ est unitaire nous avons bien:

$$
\operatorname{Res}_{x_{1}}\left(f\left(x_{1}\right), T-\Psi\left(x_{1}\right)\right)=\left(T-\Psi\left(\alpha_{1}\right)\right)=\chi_{\Psi}(T) .
$$

Montrons-le également pour $n=2$. Nous avons

$$
f_{2}\left(x_{1}, x_{2}\right)=x_{1}+x_{2}-e_{1}(\alpha)
$$

et donc

$$
\begin{aligned}
R_{1}\left(T, x_{1}\right) & =\operatorname{Res}_{x_{1}}\left(f_{1}\left(x_{1}, x_{2}\right), T-\Psi\left(x_{1}, x_{2}\right)\right) \\
& \left.=T-\Psi\left(x_{2}, e_{1}(\alpha)-x_{2}\right)\right) .
\end{aligned}
$$

D'où

$$
\begin{aligned}
R_{2}(T)= & \operatorname{Res}_{x_{2}}\left(f\left(x_{2}\right), T-\Psi\left(x_{2}, e_{1}(\alpha)-x_{2}\right)\right) \\
= & \left(T-\Psi\left(\alpha_{1}, e_{1}(\alpha)-\alpha_{1}\right)\right) \\
& \times\left(T-\Psi\left(\alpha_{2}, e_{1}(\alpha)-\alpha_{2}\right)\right) \\
= & \left(T-\Psi\left(\alpha_{1}, \alpha_{2}\right)\right)\left(T-\Psi\left(\alpha_{2}, \alpha_{1}\right)\right) \\
= & \chi_{\Psi}(T) \quad \text { d'après la formule }(3-1) .
\end{aligned}
$$

Supposons désormais que le théorème soit vrai jusqu'à $n-1$ dans tout anneau commutatif unitaire, donc en particulier dans $\mathcal{K}\left[x_{n}\right]$. Ainsi, en posant, $F(x)=f_{n-1}\left(x, x_{n}\right)=f_{n-1}(x)$ et en notant $F_{n-1}\left(x_{n-1}\right), F_{n-2}\left(x_{n-2}, x_{n-1}\right), \ldots, F_{1}\left(x_{1}, \ldots, x_{n-1}\right)$ les modules de Cauchy associés à $F$, nous avons $F_{j}\left(x_{j}, \ldots, x_{n-1}\right)=f_{j}\left(x_{j}, \ldots, x_{n}\right)$ pour $j=1, \ldots$, $n-1$. Notons $\beta_{1}\left(x_{n}\right), \ldots, \beta_{n-1}\left(x_{n}\right)$ les $n-1$ racines de $F(x)$ dans une clôture algébrique de $\mathcal{K}\left[x_{n}\right]$. Par hypothèse de récurrence et puisque $F(x)$ est unitaire, nous avons

$$
\begin{aligned}
& R_{n-1}\left(T, x_{n}\right)= \\
& \prod_{\tau \in \mathfrak{S}_{n-1}}\left(T-\Psi\left(\beta_{\tau(1)}\left(x_{n}\right), \ldots, \beta_{\tau(n-1)}\left(x_{n}\right), x_{n}\right)\right),
\end{aligned}
$$


et donc

$$
\begin{aligned}
& R_{n}(T)= \\
& \quad \prod_{i=1}^{n} \prod_{\tau \in \mathfrak{S}_{n-1}}\left(T-\Psi\left(\beta_{\tau(1)}\left(\alpha_{i}\right), \ldots, \beta_{\tau(n-1)}\left(\alpha_{i}\right), \alpha_{i}\right)\right) .
\end{aligned}
$$

Or, pour $i=1, \ldots, n$, pour $j=1, \ldots, n-1$ et pour $\tau \in \mathfrak{S}_{n-1}$, nous savons que $\beta_{\tau(j)}\left(\alpha_{i}\right)$ est une racine de

$$
F(x)=f_{2}\left(x, \alpha_{i}\right)=\frac{f\left(\alpha_{i}\right)-f(x)}{\alpha_{i}-x}
$$

et est donc une racine de $f$ distincte de $\alpha_{i}$. Ainsi, les $n$ ! $n$-uplets $\left(\beta_{\tau(1)}\left(\alpha_{i}\right), \ldots, \beta_{\tau(n-1)}\left(\alpha_{i}\right), \alpha_{i}\right)$ sur lesquels s'évalue l'invariant $\Psi$ dans le produit (4-1) sont les $n ! n$-uplets $\left(\alpha_{\sigma(1)}, \ldots, \alpha_{\sigma(n)}\right)$ où $\sigma$ parcourt $\mathfrak{S}_{n}$. Ceci achève la démonstration.

Remarque 4.8. Comme le polynôme $f$ est supposé unitaire, alors chaque module de Cauchy $f_{i}\left(x_{1}, \ldots, x_{i}\right)$ est également unitaire en $x_{i}$ pour $i=1, \ldots, n$. Dans le cas où $f$ n'est pas unitaire, et où $a$ est son coefficient dominant, il suffit de poser $g(x)=a^{n-1} f(x / a)$ qui, lui, est un polynôme unitaire. Pour garder le polynôme $f$, il faut alors introduire le coefficient dominant de $f=f_{n}$ ainsi que ceux des modules de Cauchy $f_{1}, \ldots, f_{n-1}$ dans les calculs des résultants successifs.

\section{RÉDUCTIONS AVEC DES BASES DE GRÖBNER}

\section{A. Bases de Gröbner}

L'idéal J est engendré par les $n$ polynômes

$$
e_{1}-e_{1}(\alpha), \ldots, e_{n}-e_{n}(\alpha) .
$$

Mais ces polynômes n'en forment pas une base de Gröbner ; voir [Becker et Weispfenning 1993].

Rappelons le théorème historique de Cauchy dans lequel il énonce comment les utiliser pour évaluer les polynômes symétriques :

Théorème [Cauchy 1882]. Soit $F\left(x_{1}, \ldots, x_{n}\right)$ un polynôme à coefficients dans $\mathcal{K}$ et symétrique en les variables $x_{1}, \ldots, x_{n}$. Pour éliminer $x_{n}, \ldots, x_{1}$ dans le polynôme $F$, il suffit de diviser successivement $F$ par les divers termes de la suite $f_{1}\left(x_{1}\right), \ldots, f_{n-1}\left(x_{n-1}\right)$, $f_{n}\left(x_{n}\right)$, en considérant chaque $f_{i}$ comme une fonction de $x_{i}$. Le dernier reste obtenu sera indépendant de $x_{1}, \ldots, x_{n}$ et donnera la valeur $F\left(\alpha_{1}, \ldots, \alpha_{n}\right)$ en fonction des coefficients de $f$.
Autrement dit, lorsque le polynôme $f$ est séparable, les modules de Cauchy de $f$ forment une base de Gröbner réduite de l'idéal J des relations symétriques. Pour évaluer un polynôme symétrique il suffit de le réduire modulo l'idéal J.

Notation 5.1. Pour $i \in[1, n]$, la notation $\mathcal{J}_{i}$ désigne l'idéal engendré dans $\mathcal{K}\left[x_{i}, \ldots, x_{n}\right]$ par les modules de Cauchy $f_{i}, \ldots, f_{n}$.

Il est bien connu qu'alors les polynômes $f_{i}, \ldots, f_{n}$ forment une base de Gröbner réduite pour l'ordre lexicographique de l'idéal $\mathcal{J}_{i}$ [Becker et Weispfenning 1993]. Ainsi, en considérant $\mathcal{K}\left[x_{i}, \ldots, x_{n}\right]$ comme un sous-anneau de $\mathcal{K}\left[x_{1}, \ldots, x_{n}\right]$ :

$$
\begin{aligned}
\mathcal{J}_{i} & =\mathcal{J} \cap \mathcal{K}\left[x_{i}, \ldots, x_{n}\right] \\
& =\left\{P \in \mathcal{K}\left[x_{i}, \ldots, x_{n}\right] \mid\left(\forall \sigma \in \mathfrak{S}_{n}\right)(\sigma . P)(\alpha)=0\right\} .
\end{aligned}
$$

En particulier $\mathcal{J}_{1}=\mathcal{J}$.

Le théorème de Cauchy se généralise facilement ainsi:

Théorème 5.2. Supposons que le polynôme $f$ soit réductible sur $\mathcal{K}: f=g h$ où $g, h \in \mathcal{K}[x]$ avec $\operatorname{deg}(g)=$ $m$ et $\operatorname{deg}(h)=p=n-m$. Choisissons la numérotation des racines du polynôme $f$ de telle sorte que $\alpha_{1}, \ldots, \alpha_{m}$ soient les $m$ racines du polynôme $g$. Notons $g_{1}, \ldots, g_{m}$ les modules de Cauchy du polynôme $g$ et $h_{1}, \ldots, h_{p}$ ceux du polynôme $h$. Alors les $n$ polynômes $g_{1}, \ldots, g_{m}, h_{1}, \ldots, h_{p}$ forment une base de Gröbner réduite de l'idéal $I_{\alpha}^{\mathfrak{S}_{m} \times \mathfrak{S}_{p}}$.

Démonstration. D'après [Valibouze 1999], l'idéal $I_{\alpha}^{\mathfrak{S}_{m} \times \mathfrak{S}_{p}}$ est engendré dans $\mathcal{K}\left[x_{1}, \ldots, x_{n}\right]$ par l'idéal dans $\mathcal{K}\left[x_{1}, \ldots, x_{m}\right]$ des relations symétriques entre les racines du polynôme $g$ et l'idéal dans

$$
\mathcal{K}\left[x_{m+1}, \ldots, x_{n}\right]
$$

des relations symétriques entre les racines du polynôme $h$ :

$$
\mathcal{J}_{\alpha}^{\mathfrak{S}_{m} \times \mathfrak{S}_{p}}=\mathcal{J}_{\left(\alpha_{1}, \ldots, \alpha_{m}\right)}^{\mathfrak{S}_{m}}+\mathcal{J}_{\left(\alpha_{m+1}, \ldots, \alpha_{n}\right)}^{\mathfrak{S}_{p}} .
$$

\section{B. Réduction modulo un idéal triangulaire}

Definition 5.3. Un ensemble $T$ de $n$ polynômes de $\mathcal{K}\left[x_{1}, \ldots, x_{n}\right]$ est dit triangulaire de $\mathcal{K}\left[x_{1}, \ldots, x_{n}\right]$ si $T=\left\{p_{1}\left(x_{1}\right), \ldots, p_{n}\left(x_{1}, \ldots, x_{n}\right)\right\}$ où chaque polynôme $p_{i}$ est unitaire en $x_{i}$ avec degré $\left(p_{i}, x_{i}\right)>0$.

Definition 5.4. Un idéal $\mathcal{J}$ est dit triangulaire s'il est engendré par un ensemble triangulaire. 
Exemple 5.5. L'idéal $\mathcal{J}$ est un idéal triangulaire, ainsi que l'idéal $I_{\alpha}^{\mathfrak{S}_{m} \times \mathfrak{S}_{p}}$ défini au théorème 5.2.

Soit maintenant $\mathcal{J}$ un idéal triangulaire, engendré par l'ensemble triangulaire:

$$
\left\{p_{1}\left(x_{1}\right), p_{2}\left(x_{1}, x_{2}\right), \ldots, p_{n}\left(x_{1}, \ldots, x_{n}\right)\right\} .
$$

Réduire le polynôme $\Theta$ de $\mathcal{K}\left[x_{1}, \ldots, x_{n}\right]$ par l'idéal $\mathcal{J}$ revient a réaliser successivement des divisions euclidiennes par chaque polynôme $p_{j}$ considéré comme un polynôme en $x_{j}$, pour $j \in[1, n]$. Le reste de cette division appartiendra au quotient $\mathcal{K}\left[x_{1}, \ldots, x_{n}\right] / \mathcal{J}$.

Notation 5.6. Pour $\Theta \in \mathcal{K}\left[x_{i}, \ldots, x_{n}\right][T]$ le résultat de cette réduction sera noté $\Theta \bmod \mathcal{J}$.

\section{Algorithme 5.7 (Réduction).}

entrées : $\Theta \in \mathcal{K}\left[x_{1}, \ldots, x_{n}\right][T]$

$p_{1}, \ldots, p_{n}$ : une base triangulaire de $\mathcal{J}$

sortie: $\Theta \bmod \mathcal{J}$

resultat $\leftarrow \Theta$

pour $j \in[1, \ldots, n]$ répète

resultat $\leftarrow$ Reste $\left(\right.$ resultat $\left., p_{j}, x_{j}\right)$

renvoie resultat

Où $\operatorname{Reste}\left(p, q, x_{k}\right)$ est le reste de la division euclidienne de $p$ par $q$, considérés comme des polynômes de $\mathcal{K}\left[x_{1}, \ldots, x_{k-1}, x_{k+1}, \ldots, x_{n}, T\right]\left[x_{k}\right]$.

\section{CALCUL DE LA RÉSOLVANTE ABSOLUE}

La formule (3-2) montre que le polynôme caractéristique est une puissance de la résolvante. Cette partie est donc consacrée à l'élimination de cette puissance. Le paragraphe $6 \mathrm{~A}$ rappelle les résultats de F. Lehobey sur ce point et le paragraphe 6D montre comment certaines puissances sont éliminées lorsque les calculs sont réalisés dans l'algèbre de décomposition universelle.

Les modules de Cauchy du polynôme $f$ sont notés $f_{1}, \ldots, f_{n}$.

\section{A. Méthode de Lehobey}

Dans la pratique, le calcul de la résolvante $\mathcal{L}_{\Psi, f}(T)$ ne se réalise pas sans réduire la puissance parasite due aux symétries de l'invariant $\Psi$ à chaque étape de l'algorithme 6.3 (voir (3-2)). La formation de cette puissance est illustrée au travers de l'exemple suivant :
Exemple 6.1. Fixons $n=3$ et choisissons $\Psi=2 x_{3}+$ $x_{1}+x_{2}$ un $\mathfrak{S}_{3}$-primitif $H$-invariant où $H=\mathfrak{S}_{2} \times \mathfrak{S}_{1}$. D'après la définition de la suite $\left(R_{j}\right)_{j}$ du théorème 4.7 , il vient:

$$
\begin{aligned}
& R_{n-1}\left(T, x_{3}\right)= \\
& \quad \operatorname{Res}_{x_{2}}\left(f_{2}\left(x_{2}, x_{3}\right), \operatorname{Res}_{x_{1}}\left(f_{1}\left(x_{1}, x_{2}, x_{3}\right), T-\Psi\right)\right) .
\end{aligned}
$$

Il est alors possible d'éliminer des symétries à ce niveau puisque:

$$
R_{2}\left(T, x_{3}\right)=\left(T-\left(2 x_{3}+\beta_{1}\left(x_{3}\right)+\beta_{2}\left(x_{3}\right)\right)\right)^{2},
$$

où $\beta_{1}\left(x_{3}\right)$ et $\beta_{2}\left(x_{3}\right)$ sont les racines de $f_{2}(x)$ dans $\mathcal{K}\left[x_{3}\right][x]$. En posant $V_{2}\left(T, x_{3}\right)^{2}=R_{2}\left(T, x_{3}\right)$, nous avons finalement:

$$
\mathcal{L}_{\Psi, f}(T)=\operatorname{Res}_{x_{3}}\left(f_{3}\left(x_{3}\right), V_{2}\left(T, x_{3}\right)\right) .
$$

Nous constatons que nous obtenons la résolvante au lieu de son carré donné par la formule (3-2). Cet exemple montre également qu'il est préférable d'éliminer d'abord les symétries.

L'exemple 6.1 suppose connu le polynôme $V_{2}$ qui se calcule par la méthode de F. Lehobey [1997]. Cette méthode s'appuie sur le théorème 6.2 et aboutit à l'algorithme 6.3 exposés ci-après.

Le théorème 6.2 explicite le degré de chaque puissance superflue qui apparaît après un calcul de résultant.

Théorème 6.2 (Lehobey). Soient $A$ un anneau intègre de caractéristique nulle, $f \in A[x]$ un polynôme unitaire, de degré $n ; H_{n}$ un sous-groupe de $\mathfrak{S}_{n} ; \Psi \in$ $A\left[x_{1}, \ldots, x_{n}\right]$ un $H_{n}$-invariant primitif $; f_{1}, \ldots, f_{n}$, les $n$ modules de Cauchy de $f$ et $H_{i}=\operatorname{Stab}_{\mathfrak{S}_{i}}(\Psi)$, pour $1 \leq i \leq n-1$, les $n-1$ stabilisateurs sur $\mathfrak{S}_{i}$ de $\Psi$ considéré comme un élément de

$$
A\left[x_{i+1}, \ldots, x_{n}\right]\left[x_{1}, \ldots, x_{i}\right] .
$$

Soit l'entier $m_{i}$ défini par:

$$
m_{i}=\frac{\operatorname{card} H_{i}}{\operatorname{card} H_{i-1}} \quad \text { pour } 1 \leq i \leq n,
$$

avec card $H_{0}=1$. Alors la suite finie $V_{0}, \ldots, V_{n}$ définie récursivement par $V_{0}\left(T, x_{1}, \ldots, x_{n}\right)=T-\Psi$ et

$$
\begin{aligned}
V_{i}\left(T, x_{i+1}, \ldots,\right. & \left.x_{n}\right)= \\
& \left(\operatorname{Res}_{x_{i}}\left(f_{i}\left(x_{i}\right), V_{i-1}\left(T, x_{i}, \ldots, x_{n}\right)\right)\right)^{1 / m_{i}}
\end{aligned}
$$

calcule la résolvante de $f$ par $\Psi$ :

$$
\mathcal{L}_{\Psi, f}(x)=V_{n}(x) .
$$




\section{B. La racine r-ième}

L'algorithme de calcul de la résolvante (6.3) ainsi que les algorithmes qui en découlent, font appel à la fonction racine() définie de la façon suivante:

Soient $A$ un anneau commutatif de caractéristique nulle quelconque, $m$ un entier positif et $p$ un polynôme de $\mathcal{K}\left[x_{1}, \ldots, x_{m}\right]$ unitaire en $x_{i}$, pour $i \in$ $[1 \ldots m] ;$ alors pour tout $k \in \mathbb{N}$ la fonction

$$
\operatorname{racine}\left(p^{k}, k, x_{i}\right)
$$

retourne le polynôme $p$. Cette fonction est basée sur le travail de P. Henrici [1956].

\section{C. L'algorithme de calcul de la résolvante}

\section{Algorithme 6.3 (Résolvante).}

entrées: $f:$ un polynôme de $\mathcal{K}[x]$

$\Psi$ : un polynôme de $\mathcal{K}\left[x_{1}, \ldots, x_{n}\right] \quad H$-invariant primitif

puissancesParasites : une liste d'entiers contenant les degrés des puissances parasites

sortie: la résolvante de $f$ par $\Psi$

resultat $\leftarrow T-\Psi$

pour $\mathrm{cm}$ dans modulesDeCauchy $\left(f,\left[x_{1}, \ldots, x_{n}\right]\right)$

$v$ dans $\left[x_{1}, \ldots, x_{n}\right]$

$p p$ dans puissancesParasites

répète resultat $\leftarrow$

racine (resultant $(c m$, resultat, $v), p p, T)$

renvoie resultat

Où :

- la fonction modulesDeCauchy $\left(f,\left[x_{1}, \ldots, x_{n}\right]\right)$ renvoie la liste $\left[f_{1}\left(x_{1}\right), \ldots, f_{n}\left(x_{n}\right)\right]$ des modules de Cauchy de $f$;

- racine $(f, n, y)$ calcule la racine $n$-ième de $f$ comme polynôme en $y$ (voir section $6 \mathrm{~B}$ );

- la liste des puissances parasites puissancesParasites est fonction de l'invariant. Elle peut être calculée en utilisant des stabilisateurs (voir théorème 6.2) avec le logiciel GAP [Schönert et al. 1995] ou par la factorisation des calculs intermédiaires.

Démonstration. Cet algorithme découle des théorèmes 4.7 et 6.2 .

Exemple 6.4. Prenons le polynôme

$$
f=x^{6}-243 x^{2}+729
$$

et l'invariant $\Psi=x_{5} x_{6}+x_{3} x_{4}+x_{1} x_{2}$. Le temps nécessaire au calcul du polynôme caractéristique puis à celui de la résolvante à partir de la formule (3-2) est de 1 heure et 40 minutes. Avec l'algorithme 6.3 le temps nécessaire au calcul de la résolvante n'est plus que de 1 seconde.

\section{D. Calculs modulo l'idéal des relations symétriques}

L'un des inconvénients de l'algorithme 6.3 est la croissance des degrés en les variables $x_{1}, \ldots, x_{n}$ dans les calculs intermédiaires du polynôme resultat. Nous étudions donc le moyen de contrôler cette croissance par réductions utilisant des divisions euclidiennes. Nous constaterons que la méthode employée aboutit parfois à l'élimination de plusieurs variables lors d'un même pas de boucle. L'élimination prématurée des variables par réduction n'occasionne donc aucune puissance superflue. Une réduction est alors équivalente à autant de pas de l'algorithme 6.3 que de variables éliminées prématurément par réduction.

Réduction de polynômes. Soit $i \in[1, n]$. Puisque $f_{i}$, $\ldots, f_{n}$ est une base de Gröbner réduite de l'idéal $\mathcal{J}_{i}$ de dimension zéro (voir notation 5.1), l'anneau quotient $\mathcal{K}\left[x_{i}, \ldots, x_{n}\right] / \mathcal{J}_{i}$ est engendré en tant que $\mathcal{K}$ module par les monômes $m$ tels que, pour $j \in[i, n]$, degré $\left(m, x_{j}\right)<j=\operatorname{deg}_{x_{j}}\left(f_{j}\left(x_{j}\right)\right.$ ) (voir définition 2.12). Autrement dit:

$$
\mathcal{K}\left[x_{i}, \ldots, x_{n}\right] / \mathcal{J}_{i} \simeq \bigoplus_{0 \leq b_{j}<j} \mathcal{K} \cdot x_{i}^{b_{i}} \ldots x_{n}^{b n} .
$$

Ainsi les degrés en $x_{j}(0<j \leq n)$ des polynômes de l'algorithme 6.3 peuvent être contrôlés pour ne pas dépasser $j-1$.

Notation 6.5. Soit $I$ un idéal quelconque. La notation

\section{expression mod $I$,}

qui désigne le résultat de la réduction de expression modulo l'idéal $I$, sous-entend que tous les calculs d'expression sont effectués modulo l'idéal $I$.

Élimination automatique des puissances superflues. Commençons par des exemples qui illustrent comment, après une réduction, des variables peuvent être éliminées prématurément évitant ainsi, d'une part, des 
calculs de résultants et, d'autre part, l'apparition de puissances superflues.

Exemple 6.6. L'arité de l'invariant est importante, ce que l'on peut voir de la façon suivante: Prenons un polynôme $f$ de degré 4 . Supposons que $\Psi=x_{4}+2 x_{3}$ où 2 est donc l'arité de $\Psi$ (voir définition 2.1). Soit $S_{0}=(T-\Psi) \bmod \mathcal{J}=T-\left(x_{4}+2 x_{3}\right)$, puis $S_{1}=$ $\operatorname{Res}_{x_{3}}\left(f_{3}\left(x_{3}, x_{4}\right), S_{0}\right)=T^{3}+\left(2-x_{4}\right) T^{2}+T\left(3 x_{4}^{2}\right)+$ $5 x_{4}^{3}+6 x_{4}^{2}=S_{1} \bmod \mathcal{J}_{4}$. Alors

$$
\begin{aligned}
S_{2}= & \operatorname{Res}_{x_{4}}\left(f_{4}\left(x_{4}\right), S_{1}\right) \\
= & T^{12}+9 T^{11}+33 t^{10}+63 T^{9}+40 T^{8} \\
& \quad-120 T^{7}-346 T^{6}-336 T^{5}+2348 T^{4} \\
& \quad+7332 T^{3}+7416 T^{2}+3888 T+5864 \\
& \quad \mathcal{L}_{\Psi, f} .
\end{aligned}
$$

Le calcul de la résolvante s'est alors réalisé en évitant deux résultants et par conséquent sans l'apparition de puissances superflues induites par ces résultants. De manière générale, si $m \leq 1$ et $\Psi \in \mathcal{K}\left[x_{m}, \ldots, x_{n}\right]$ est un $\mathfrak{S}_{n-m}$-primitif $K$-invariant alors le calcul de la résolvante se réalise sans les $m$ premiers résultants définissant la suite $\left(R_{j}\right)_{j}$ du théorème 4.7. Ces premiers résultants sont nécessaires au calcul du polynôme caractéristique mais parfaitement inutiles à celui de la résolvante. De plus, ils introduisent une puissance superflue égale à $m$ !. En effet, si la suite $\left(R_{j}\right)_{j}$ du théorème 4.7 est modifiée en ignorant les $m$ premiers termes et en posant

$$
R_{m+1}=T-\Psi\left(x_{m}, \ldots, x_{n}\right),
$$

alors

$$
\chi_{\Psi}=R_{n}^{m !} \quad \text { et } \quad R_{n}=\left(\mathcal{L}_{\Psi, f}\right)^{\operatorname{card}(K)} .
$$

Exemple 6.7. Prenons $f=x^{4}+x^{3}+2$ et choisissons $\Psi=x_{3} x_{4}+x_{1} x_{2}$ un $\mathcal{D}_{4}$-invariant primitif (où $\mathcal{D}_{4}$ est le groupe diédral). Le calcul du polynôme

$$
S_{0}=(T-\Psi) \bmod \mathcal{J}_{1}
$$

aboutit à $S_{0}=x_{3}^{2}+\left(-2 x_{4}-1\right) x_{3}-x_{4}^{2}-x_{4}+T$. Les variables $x_{1}$ et $x_{2}$ sont éliminées sans un calcul de résultant. Le calcul du polynôme

$$
S_{1}=\operatorname{Res}_{x_{3}}\left(f_{3}\left(x_{3}\right), S_{0}\right) \bmod \mathcal{J}_{4}
$$

aboutit à $S_{1}=T^{3}-8 T-2$. Comme le degré du polynôme $S_{1}$ est égal à celui de la résolvante $\mathcal{L}_{\Psi, f}$, le théorème 6.8 nous assure que ces polynômes sont égaux.
Théorème 6.8. Posons $I_{n+1}=(0)$ et définissons la suites finie d'entiers $\left(k_{i}\right)_{0 \leq i \leq j}\left(k_{i} \in[0, n]\right)$ et la suite finie de polynômes $\left(S_{i}\right)_{0 \leq i \leq j}$ de la manière suivante:

- l'entier naturel $n-k_{i}+1$ est l'arité du polynôme $S_{i}$ qui appartient à $\mathcal{K}[T]\left[x_{k_{i}}, \ldots, x_{n}\right]$;

- l'entier $j$ est le plus petit entier $i$ tel que $k_{i}=n+1$ (i.e l'arité de $S_{i}$ est nulle);

- Au départ:

$$
S_{0}\left(T, x_{k_{0}}, \ldots, x_{n}\right)=T-\Psi \bmod \mathcal{J}_{1}
$$

et pour $i \in[0, j[$

$S_{i+1}\left(T, x_{k_{(i+1)}}, \ldots, x_{n}\right)=$

$\operatorname{Res}_{x_{k_{i}}}\left(f_{k_{i}}\left(x_{k_{i}}\right), S_{i}\left(T, x_{k_{i}}, \ldots, x_{n}\right)\right)^{1 / m_{k_{i}}} \bmod \mathcal{J}_{k_{i}+1}$,

où l'entier $m_{k_{i}}$ est celui défini au théorème 6.2.

Alors le dernier terme de la suite est une puissance de la résolvante cherchée:

$$
S_{j}(T)^{m}=\mathcal{L}_{\Psi, f}(T) \quad \text { pour } m>0 .
$$

Démonstration. La démonstration se déroule en trois étapes. La première montre que l'on peut remplacer chaque $V_{i}$ de la suite $\left(V_{i}\right)_{i}$ définie au théorème 6.2 par $V_{i} \bmod \mathcal{J}_{i+1}$. La seconde montre que l'on peut réaliser tous les calculs de la suite $\left(V_{i}\right)_{i}$ modulo les idéaux $\mathcal{J}_{j}(j \in[1, n])$ et la troisième étape montre comment éviter des calculs en tenant compte des variables éliminées prématurément.

Soit $W=V_{i} \bmod \mathcal{J}_{i+1}$, c'est à dire, d'après l'algorithme $6.10, V_{i}=\sum_{j=i+1}^{n} \$ f_{j}\left(x_{j}\right)+W$ où $\$$ désigne un polynôme quelconque (en l'occurrence un quotient d'une division euclidienne par $\left.f_{j}\left(x_{j}\right)\right)$. Alors dans les résultants successifs définissant $V_{i+1}, \ldots, V_{n}$, le polynôme $V_{i}$ peut-être remplacé par le polynôme $W$ sans que le polynôme $V_{n}$ soit modifié, puisque ces résultants sont réalisés avec les polynômes $f_{i+1}\left(x_{i+1}\right)$, $\ldots, f_{n}\left(x_{n}\right)$.

Maintenant posons $P=\operatorname{Res}_{x_{i}}\left(f_{i}\left(x_{i}\right), V_{i-1}\right)=V_{i}^{m_{i}}$ et $S=P \bmod \mathcal{J}_{i+1}$, c'est à dire, d'après l'algorithme $6.10, P=\sum_{j=i+1}^{n} \$ f_{j}\left(x_{j}\right)+S$. Alors $S=W^{m_{i}} \bmod$ $\mathcal{J}_{i+1}$. Ainsi, en remplaçant $V_{i}$ par $S^{1 / m_{i}} \bmod \mathcal{J}_{i+1}$ dans la suite $\left(V_{j}\right)_{j}$, le résultat $V_{n}=\mathcal{L}_{\Psi, f}$ n'est pas modifié.

Tenons compte maintenant des variables de $W$. Supposons que le polynôme $V_{i}$ soit remplacé par $W$ dans la suite $\left(V_{j}\right)_{j}$. Nous avons donc

$$
V_{i+1}^{m_{i+1}}=\operatorname{Res}_{x_{i+1}}\left(f_{i+1}\left(x_{i+1}\right), W\right) .
$$


Si $W$ ne dépend pas de $x_{i+1}$, alors $V_{i+1}^{m_{i+1}}=W^{i+1}$. Ainsi, si $V_{i+1}$ est remplacé par $W$ dans la suite $\left(V_{j}\right)_{j}$, alors $V_{n}=\mathcal{L}_{\Psi, f}^{i+1 / m_{i+1}}$.

Remarque 6.9. Dans l'équation (6-1), l'entier $m$ sera égal à 1 si les résultants évités par élimination prématurée des variables (voir la dernière partie de la démonstration) correspondent à des symétries de l'invariant $\Psi$ et non pas au fait que l'invariant $\Psi$ n'est pas séparable. Par exemple, si $\Psi$ est un $H$ invariant primitif, où $H$ est un sous-groupe propre de $\mathfrak{S}_{n}$, et que $\Psi-\Psi(\alpha) \in \mathcal{J}$, alors $i_{0}=0, S_{i_{0}}=$ $T-\Psi(\alpha) \in \mathcal{K}[T]$ et $\mathcal{L}_{\Psi, f}=S_{i_{0}}^{\left[\mathfrak{S}_{n}: H\right]}$. L'identification du groupe de Galois du polynôme $f$ se réalise avec les facteurs simples des résolvantes. Donc, en pratique, dès que le programme détecte que le saut de plusieurs résultants n'aboutira pas au calcul exact de la résolvante (i.e. $m=1$ ), il sera stoppé pour choisir un autre invariant du même groupe ou pour transformer le polynôme $f$. Cette détection se fait avec les degrés des modules de Cauchy et les entiers $m_{i}$ de Lehobey dès que le calcul d'un résultant est évité.

\section{Algorithme 6.10 (Calcul de la résolvante avec réductions).}

entrées: $f$ : un polynôme de $\mathcal{K}[x]$,

$\Psi$ : un polynôme de $\mathcal{K}\left[x_{1}, \ldots, x_{n}\right] \quad H$-invariant primitif

puissancesParasites : une liste d'entiers contenant les degrés des puissances parasites

sortie: la $H$-résolvante de $f$ par $\Psi$

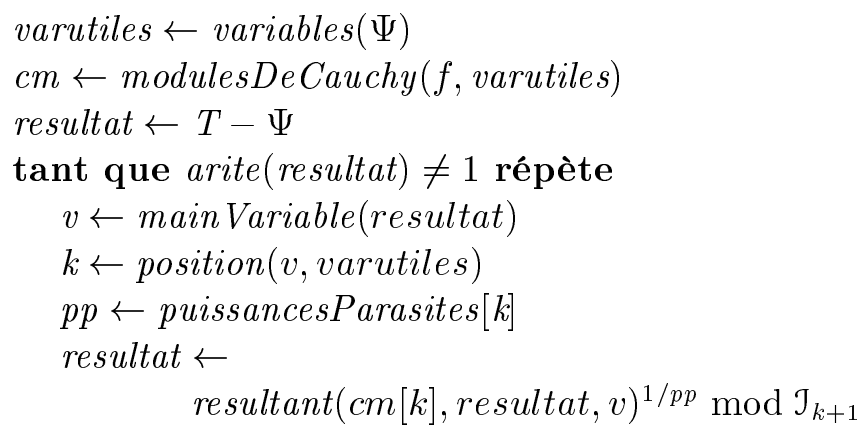

Où :

- la fonction arite $(P)$ renvoie l'arité de $P$,

- la fonction position $(v, l v)$ renvoie la position de $v$ dans la liste $l v$,

- la fonction main Variable $(P)$ renvoie la variable principale de $P$,
- la notation $l[k]$ désigne le $k$-ième élément de la liste $l$,

- la fonction variables $(P)$ renvoie la liste des variables de $P$,

- la fonction modulesDeCauchy $\left(f,\left[x_{1}, \ldots, x_{n}\right]\right)$ renvoie la liste $\left[f_{1}\left(x_{1}\right), \ldots, f_{n}\left(x_{n}\right)\right]$ des modules de Cauchy de $f$,

- l'idéal $\mathcal{J}_{k+1}$ est défini notation 5.1,

- la notation $P \bmod \mathcal{J}_{k+1}$ est décrite en notation 6.5 ,

- le calcul de $P^{\frac{1}{k}} \bmod \mathcal{J}$ est réalisé à l'aide de la fonction racine $(p, k, v)$ décrite au paragraphe $6 \mathrm{~B}$.

- La liste des puissances parasites puissancesParasites est fonction de l'invariant. Elle peut être calculée en utilisant des stabilisateurs (voir théorème 6.2) avec le logiciel GAP [Schönert et al. 1995] ou par la factorisation des calculs intermédiaires.

\section{GÉNÉRALISATION AUX MULTI-RÉSOLVANTES}

Soient $F=\left(f^{(1)}, \ldots, f^{(p)}\right)$ un $p$-uplet de polynômes de $\mathcal{K}[x]$ de degrés respectifs $d_{1}, \ldots, d_{p}$ et $d=$ $d_{1}+\cdots+d_{p}$. Donnons-nous $x_{1}, \ldots, x_{d}$ des indéterminées. Soit $\Omega_{F}$ la liste des racines des polynômes de $F$, ordonnées ainsi : $\Omega_{F}=\left(\Omega^{(1)}, \ldots, \Omega^{(p)}\right)$ où $\Omega^{(i)}$ est une liste quelconque des racines du polynôme $f^{(i)}$ pour $i \in[1, p]$.

Fixons $\Psi \in \mathcal{K}\left[x_{1}, \ldots, x_{d}\right]$ et posons $\mathcal{S}=\mathfrak{S}_{d_{1}} \times$ $\cdots \times \mathfrak{S}_{d_{p}}$.

Definition 7.1. La multi-résolvante absolue de $F$ par $\Psi$, notée $\mathcal{L}_{\Psi, F}$, est la résolvante relative $\mathcal{L}_{\Psi, s, \Omega_{F}}$. La multi-résolvante de $F$ est donc le polynôme

$$
\mathcal{L}_{\Psi, F}=\prod_{\Theta \in \delta . \Psi}\left(y-\Theta\left(\Omega_{F}\right)\right)
$$

Pour calculer une multi-résolvante, il suffit d'utiliser les algorithmes de calcul des résolvantes conçus pour des invariants avec des coefficients non numériques. Dans la pratique, le corps $\mathcal{K}$ peut être remplacé par un anneau intègre de caractéristique nulle (voir théorème 6.2). En ce cas l'invariant $\Psi$ est considéré successivement comme un polynôme en $\left(x_{1}, \ldots, x_{d_{1}}\right)$, puis en $\left(x_{d_{1}+1}, \ldots, x_{d_{1}+d_{2}}\right), \ldots$, puis 
enfin en $\left(x_{d_{1}+\cdots+d_{p-1}+1}, \ldots, x_{d}\right)$. L'algorithme de calcul d'une multi-résolvante est donc le suivant.

\section{Algorithme 7.2 (Multi-résolvante absolue).}

entrées: $F=\left[f^{(1)}, \ldots, f^{(p)}\right]$ : une liste de polynômes de $\mathcal{K}[x]$

$\Psi$ : un polynôme de $\mathcal{K}\left[x_{1}, \ldots, x_{n}\right]$ qui est $H$ invariant primitif

$l p p:$ la liste des degrés des puissances parasites

lvar: la liste des variables $x_{1}, \ldots, x_{n}$

sortie: la multi-résolvante de $F$ par $\Psi$

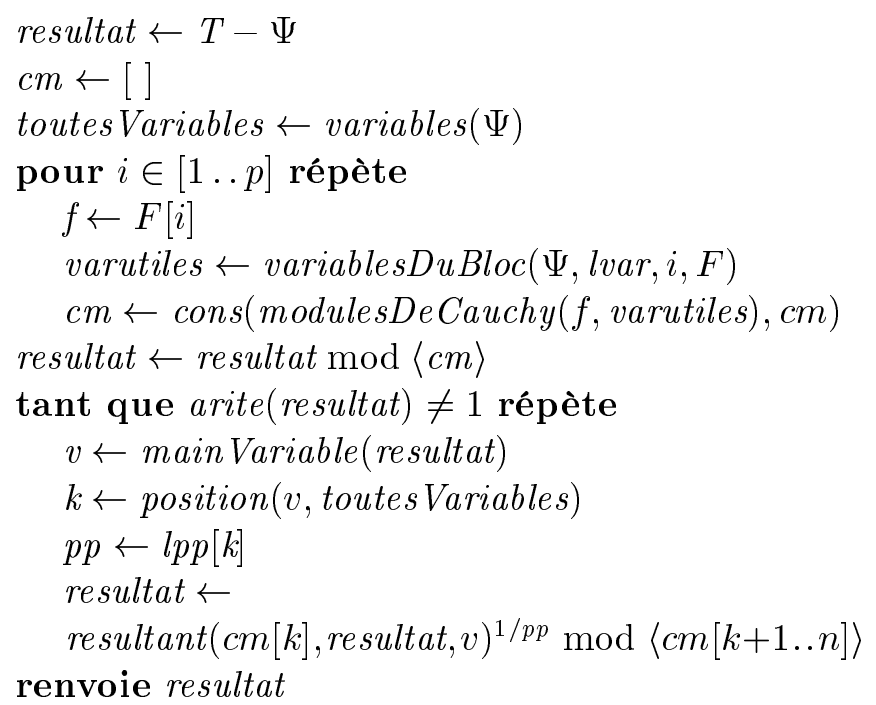

Où:

- variablesDuBloc $(\Psi$, lvar, $i, F)$ renvoie les variables du polynôme $\Psi$ associées au polynôme $F[i]$,

- la fonction $\operatorname{arite}(P)$ renvoie l'arité du polynôme $P$,

- la fonction position $(v, l v)$ renvoie la position de l'élément $v$ dans la liste $l v$,

- la fonction main Variable $(P)$ renvoie la variable principale du polynôme $P$,

- la notation $l[k]$ désigne le $k$-ième élément de la liste $l$,

- la notation $l[k \ldots j]$ désigne les éléments $k, k+1$, $\ldots, j$ de la liste $l$,

- la fonction cons $(e, l)$ renvoie la liste composée de l'élément $e$ et de la liste $l$,

- la fonction variables $(P)$ renvoie la liste des variables du polynôme $P$,

- la fonction modulesDeCauchy $\left(f,\left[x_{1}, \ldots, x_{n}\right]\right)$ renvoie la liste $\left[f_{1}\left(x_{1}\right), \ldots, f_{n}\left(x_{n}\right)\right]$ des modules de Cauchy du polynôme $f$,
- la notation $\langle\mathrm{cm}\rangle$ désigne l'idéal engendré par les polynômes de $\mathrm{cm}$,

- l'expression $P \bmod \mathcal{J}$ qui désigne la réduction du polynôme $P$ modulo l'idéal J est décrite en notation 6.5 ,

- le calcul de $P^{1 / k} \bmod \mathcal{J}$ est réalisé à l'aide de la fonction racine $(p, k, v)$ décrite au paragraphe $6 \mathrm{~B}$.

Voyons sur un exemple comment ce calcul est réalisé avec la méthode proposée dans cet article:

Exemple 7.3. Prenons les polynômes $f=x^{2}+5 x-89$ et $g=x^{2}-9 x-23$ et l'invariant $\Psi=x_{1} x_{3}+x_{2} x_{4}^{3}$.

Soient $f_{1}\left(x_{1}, x_{2}\right)=x_{2}+x_{1}-9$ et $f_{2}\left(x_{1}\right)=x_{1}^{2}-$ $9 x_{1}-23$, les modules de Cauchy associés au polynôme $f$. Soient $g_{1}\left(x_{3}, x_{4}\right)=x_{4}+x_{3}+5$ et $g_{2}\left(x_{3}\right)=$ $x_{3}^{2}+5 x_{3}-89$, les modules de Cauchy associés au polynôme $g$. Nous avons la liste $\mathrm{cm}=\left[g_{1}, g_{2}, f_{1}, f_{2}\right]$. Calculons la suite $\left(R_{i}\right)_{i}$ correspondant à la suite des valeurs prises par la variable resultat de l'algorithme 7.2 .

$$
\begin{aligned}
R_{0}= & T-\Psi \bmod \langle c m\rangle \\
= & \left(-115 x_{1}+1026\right) x_{3}-1015 x_{1}+T+9135, \\
R_{1}= & \operatorname{resultant}_{x_{3}}\left(R_{0}, g_{2}\right) \bmod \left\langle f_{1}, f_{2}\right\rangle \\
= & T^{2} \quad+(-1455 T+6343920) x_{1} \\
& \quad+T+13140 T-73902264, \\
R_{2}= & \text { resultant }_{x_{1}}\left(R_{1}, f_{2}\right) \\
= & T^{4} \quad+13185 T^{3}-138809523 T^{2} \\
& \quad+200429193960 T+316431786384576 .
\end{aligned}
$$

Alors $R_{2}=\mathcal{L}_{\Psi,(f, g)}$.

\section{IMPLANTATION}

Le système de calcul formel Axiom [Jenks et Sutor 1992] a été choisi pour l'implantation des différents algorithmes. Ce choix est motivé par la grande modularité d'Axiom, parfaitement adaptée aux mathématiques. L'implantation se veut aussi générale que possible. Ainsi le choix du domaine de polynômes est laissé libre à l'utilisateur des différents programmes.

\section{A. L'organisation générale}

La figure 1 décrit l'organisation des différents $p a$ quets (packages), domaines (domains) et catégories (categories). 


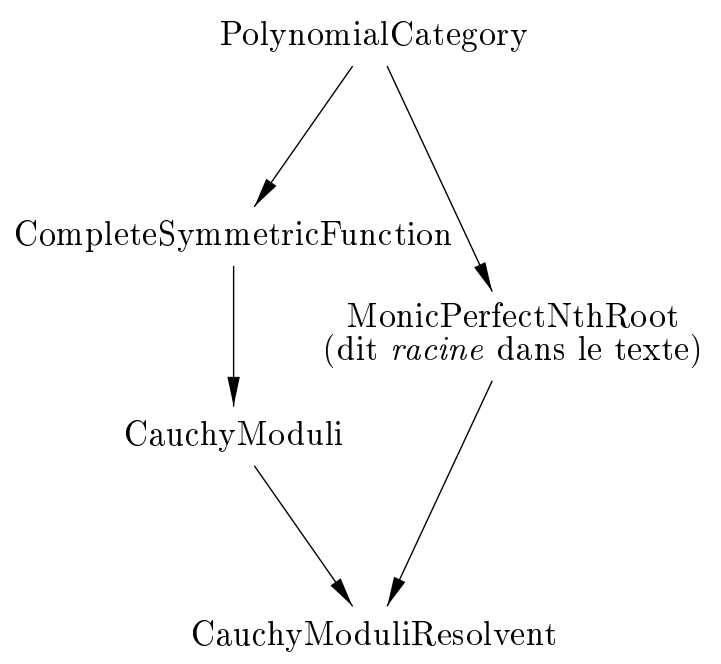

FIGURE 1. Organisation du logiciel.

\section{B. Un exemple commenté}

Voici comment en Axiom obtenir la résolvante $\mathcal{L}_{\Theta, f}$ comme polynôme en la variable $y$, où $f=$ $x^{5}+4 x^{4}-17 x^{3}-x^{2}+x+1$ et $\Theta=x_{4} x_{5}^{2}+x_{3} x_{4}^{2}+$ $x_{2} x_{3}^{2}+x_{5} x_{1}^{2}+x_{1} x_{2}^{2}$.
Définition des domaines utilisés. La variable $\mathrm{R}$ représente l'anneau de base (dans ce cas $\mathbb{Z})$ :

\section{$\mathrm{R}:=$ Integer}

La variable POL représente l'anneau de polynôme $\mathrm{R}[x]$ (ici $\mathbb{Z}[x]$ ). La variable $\mathrm{x}$ est définie comme le polynôme $x$ :

POL:=UnivariatePolynomial $(x, R)$

$\mathrm{x}: \mathrm{POL}:=\mathrm{x}$

Le choix de la représentation des polynômes de $\mathrm{R}\left[x_{1}, \ldots, x_{n}, y\right]$ est relativement vaste. Des tests ont montré que les performances varient de façon significative selon l'implantation choisie. La représentation récursive des polynômes est particulièrement adaptée aux algorithmes 6.3 et 6.10 . La variable OVL désigne le domaine des variables de l'anneau de polynôme $\mathrm{R}\left[x_{1}, \ldots, x_{n}, y\right]$. La variable DP désigne l'anneau de polynômes $\mathrm{R}\left[x_{1}, \ldots, x_{6}, y\right]$ :

lv: $=[\mathrm{x} 6, \mathrm{x} 5, \mathrm{x} 4, \mathrm{x} 3, \mathrm{x} 2, \mathrm{x} 1, \mathrm{y}]$

OVL: $=$ OrderedVariableList $(\mathrm{lv})$

DP : =SparseMultivariatePolynomial (R, OVL)

Les variables $\mathrm{x} 1, \ldots, \mathrm{x} 6$ représentent les polynômes $x_{1}, \ldots, x_{6}$ de DP :

$$
\begin{aligned}
& \mathrm{x} 1 \text { :=monomial (1\$DP, variable (x1) \$(OVL)::(OVL) , 1) \$DP; } \\
& \mathrm{x} 2:=\text { monomial (1 } \$ D P \text {, variable (x2) } \$(\text { OVL) :: (OVL) , 1) \$DP; } \\
& \mathrm{x} 3:=\text { monomial (1 } \$ D P \text {, variable (x3) \$ (OVL):: (OVL) , 1) \$DP; } \\
& \mathrm{x} 4:=\text { monomial (1\$DP, variable (x4) \$ (OVL):: (OVL) , 1) \$DP; } \\
& \mathrm{x} 5:=\text { monomial (1 } \$ \mathrm{DP} \text {, variable (x5) \$ (OVL)::(OVL) , 1) \$DP; } \\
& \mathrm{x} 6:=\text { monomial (1\$DP, variable (x6) \$(OVL)::(OVL) , 1) \$DP; }
\end{aligned}
$$

Instanciation du paquet principal. La variable DRES représente une instance du paquet CauchyModuliResolvent paramétré par les types définis précédemment. La signature du paquet CauchyModuliResolvent est la suivante :

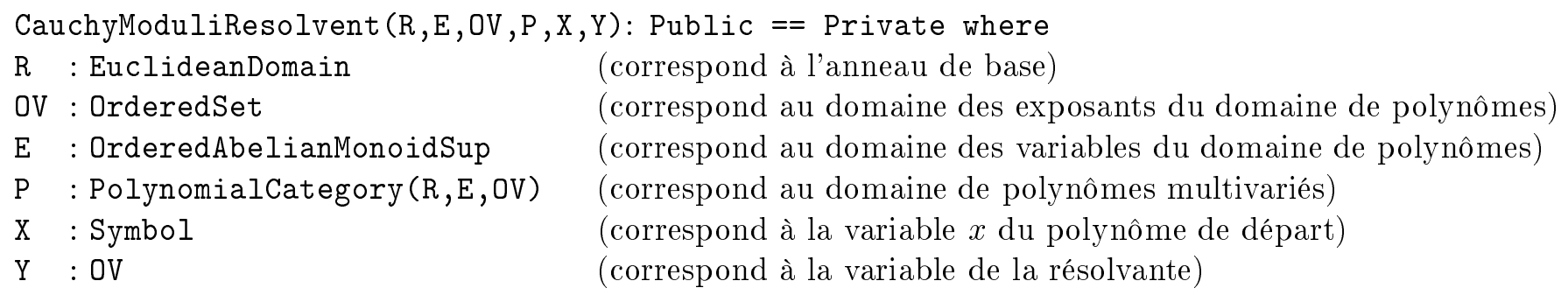

Utilisation du paquet. La variable thetaG1 représente l'invariant $\Theta$; la variable $f$ représente le polynôme $f$; la variable lpp représente la liste des puissances parasites (voir théorème 6.2). 


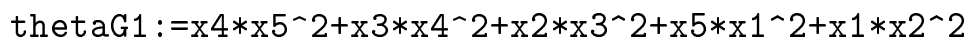

$f:=x \wedge 5+4 * x \wedge 4-17 * x \wedge 3-x \wedge 2+x+1$

lpp : $=[1,1,1,1,1,5]$

La résolvante de $f$ par thetaG1 s'obtient par la commande suivante:

$r 1:=$ resolvent $(f$, thetaG $1,1 \mathrm{pp})$ \$DRES

Le résultat est le suivant:

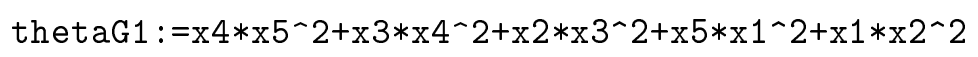

(106) $x^{2} x^{2}+x^{2} \times x^{2}+x^{2}+x^{2}+x^{2}+x 1 x^{2}$

Type: SparseMultivariatePolynomial (Integer, OrderedVariableList $[\mathrm{x} 6, \mathrm{x} 5, \mathrm{x} 4, \mathrm{x} 3, \mathrm{x} 2, \mathrm{x} 1, \mathrm{y}]$ ) Time: $0.05($ IN $)+0.02($ OT $)=0.07 \mathrm{sec}$

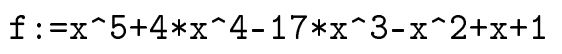

$5 \quad 4 \quad 3 \quad 2$

(107) $\mathrm{x}+4 \mathrm{x}-17 \mathrm{x}-\mathrm{x}+\mathrm{x}+1$

Type: UnivariatePolynomial (x, Integer)

Time: $0.03($ IN $)=0.03 \mathrm{sec}$

$r 1:=$ resolvent $(f$, thetaG1 $[1,1,1,1,1,5])$ \$DRES

(108)

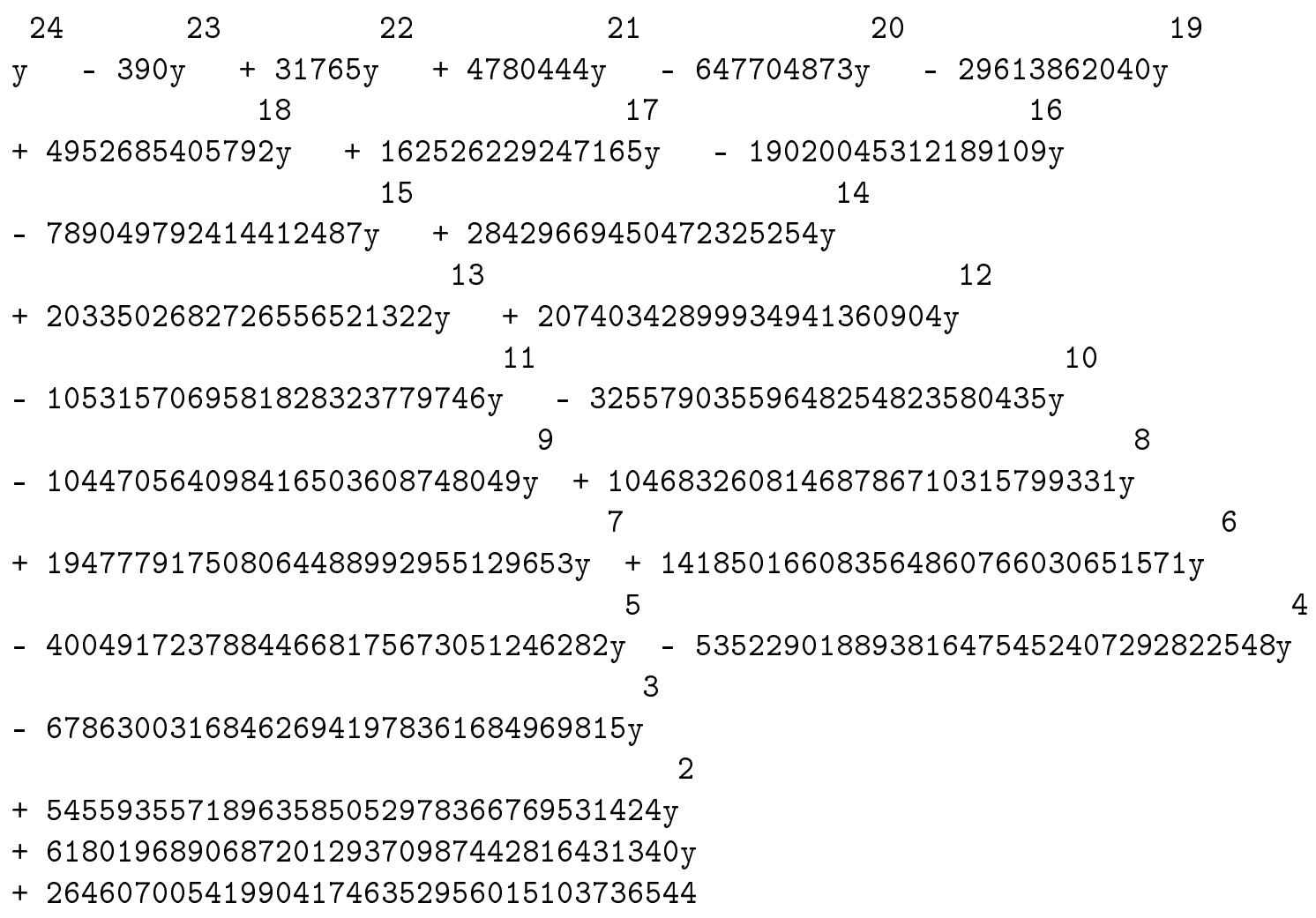

Type: SparseMultivariatePolynomial (Integer, OrderedVariableList $[\mathrm{x} 6, \mathrm{x} 5, \mathrm{x} 4, \mathrm{x} 3, \mathrm{x} 2, \mathrm{x} 1, \mathrm{y}]$ ) Time: $0.02($ IN $)+27.36(\mathrm{EV})+0.07(\mathrm{OT})+9.32(\mathrm{GC})=36.76 \mathrm{sec}$ 


\section{TEMPS DE CALCULS ET COMPARAISONS AVEC SYM}

Nous comparons maintenant les différents algorithmes de l'article et le logiciel SYM |Valibouze 1989] qui intègre différents algorithmes de calcul de résolvantes dont un algorithme général basé sur le calcul des fonctions puissances des racines de la résolvante. Lorsque la forme de l'invariant induit des formules closes de ces fonctions puissances, le logiciel SYM offre des algorithmes spécialisés. SYM est une extension du système de calcul formel Maxima $[$ Maxima $\geq 1999]$.

Les temps donnés correspondent aux temps "machine" donnés par les différents logiciels. Ils ont été mesurés sur un Pentium Pro à 200 Mhz doté de 512 Mo de mémoire vive.

\begin{tabular}{|c|c|c|}
\hline $\mathrm{L}$ & $\mathrm{H}$ & L-primitif $\mathrm{H}$-invariant \\
\hline $\mathfrak{S}_{6}$ & $T_{1}$ & $x_{5} x_{6}^{2}+x_{4} x_{5}^{2}+x_{3} x_{4}^{2}+x_{2} x_{3}^{2}+x_{6} x_{1}^{2}+x_{1} x_{2}^{2}$ \\
\hline $\mathfrak{S}_{6}$ & $T_{2}$ & $\begin{array}{l}\left(x_{5} x_{6}+x_{3} x_{4}+x_{1} x_{2}\right)+2\left(x_{4} x_{6}+x_{3} x_{5}+x_{2} x_{6}+x_{2} x_{4}+x_{1} x_{5}+x_{1} x_{3}\right)+3\left(x_{4} x_{5}+\right. \\
\left.x_{2} x_{3}+x_{1} x_{6}\right)\end{array}$ \\
\hline $\mathfrak{S}_{6}$ & $T_{3}$ & $\left(x_{5} x_{6}+x_{4} x_{5}+x_{3} x_{4}+x_{2} x_{3}+x_{1} x_{6}+x_{1} x_{2}\right)$ \\
\hline $\mathfrak{S}_{6}$ & $T_{4}$ & $\begin{array}{l}\left(x_{6} x_{5}^{2}+x_{4} x_{6}^{2}+x_{5} x_{4}^{2}+x_{3} x_{5}^{2}+x_{4} x_{3}^{2}+x_{6} x_{2}^{2}+x_{2} x_{4}^{2}+x_{3} x_{2}^{2}+x_{1} x_{6}^{2}+x_{5} x_{1}^{2}+x_{1} x_{3}^{2}+\right. \\
\left.x_{2} x_{1}^{2}\right)+2\left(x_{3} x_{5} x_{6}+x_{2} x_{4} x_{5}+x_{2} x_{3} x_{6}+x_{1} x_{4} x_{6}+x_{1} x_{3} x_{4}+x_{1} x_{2} x_{5}\right)+3\left(x_{3} x_{6}^{2}+\right. \\
\left.x_{6} x_{3}^{2}+x_{2} x_{5}^{2}+x_{5} x_{2}^{2}+x_{1} x_{4}^{2}+x_{4} x_{1}^{2}\right)+4\left(x_{3} x_{4} x_{6}+x_{2} x_{5} x_{6}+x_{2} x_{3} x_{5}+x_{1} x_{4} x_{5}+\right. \\
\left.x_{1} x_{3} x_{6}+x_{1} x_{2} x_{4}\right)+5\left(x_{3} x_{4} x_{5}+x_{2} x_{4} x_{6}+x_{1} x_{5} x_{6}+x_{1} x_{2} x_{3}\right)\end{array}$ \\
\hline $\mathfrak{S}_{6}$ & $T_{5}$ & $x_{4} x_{6}^{2}+x_{3} x_{5}^{2}+x_{6} x_{2}^{2}+x_{2} x_{4}^{2}+x_{5} x_{1}^{2}+x_{1} x_{3}^{2}$ \\
\hline $\mathfrak{S}_{6}$ & $T_{6}$ & $x_{3} x_{5} x_{6}+x_{2} x_{4} x_{5}+x_{2} x_{3} x_{6}+x_{1} x_{4} x_{6}+x_{1} x_{3} x_{4}+x_{1} x_{2} x_{5}$ \\
\hline $\mathfrak{S}_{6}$ & $T_{12}$ & $\begin{array}{l}x_{3} x_{5} x_{6}+x_{3} x_{4} x_{6}+x_{2} x_{5} x_{6}+x_{2} x_{4} x_{5}+x_{2} x_{3} x_{4}+x_{1} x_{4} x_{6}+x_{1} x_{4} x_{5}+x_{1} x_{3} x_{5}+ \\
x_{1} x_{2} x_{6}+x_{1} x_{2} x_{3}\end{array}$ \\
\hline $\mathfrak{S}_{6}$ & $T_{14}$ & $\begin{array}{l}x_{3} x_{5} x_{4} x_{6}\left(x_{3} x_{5}+x_{4} x_{6}\right)+x_{2} x_{4} x_{5} x_{6}\left(x_{2} x_{4}+x_{5} x_{6}\right)+x_{4} x_{5} x_{1} x_{6}\left(x_{4} x_{5}+x_{1} x_{6}\right)+ \\
x_{2} x_{5} x_{3} x_{6}\left(x_{2} x_{5}+x_{3} x_{6}\right)+x_{1} x_{3} x_{5} x_{6}\left(x_{1} x_{3}+x_{5} x_{6}\right)+x_{2} x_{6} x_{1} x_{5}\left(x_{2} x_{6}+x_{1} x_{5}\right)+ \\
x_{3} x_{4} x_{2} x_{6}\left(x_{3} x_{4}+x_{2} x_{6}\right)+x_{1} x_{4} x_{3} x_{6}\left(x_{1} x_{4}+x_{3} x_{6}\right)+x_{1} x_{2} x_{4} x_{6}\left(x_{1} x_{2}+x_{4} x_{6}\right)+ \\
x_{2} x_{3} x_{1} x_{6}\left(x_{2} x_{3}+x_{1} x_{6}\right)+x_{2} x_{3} x_{4} x_{5}\left(x_{2} x_{3}+x_{4} x_{5}\right)+x_{3} x_{4} x_{1} x_{5}\left(x_{3} x_{4}+x_{1} x_{5}\right)+ \\
x_{1} x_{4} x_{2} x_{5}\left(x_{1} x_{4}+x_{2} x_{5}\right)+x_{1} x_{2} x_{3} x_{5}\left(x_{1} x_{2}+x_{3} x_{5}\right)+x_{1} x_{3} x_{2} x_{4}\left(x_{1} x_{3}+x_{2} x_{4}\right)\end{array}$ \\
\hline $\mathfrak{S}_{6}$ & $\mathfrak{S}_{2} \times \mathfrak{S}_{2} \times \mathfrak{S}_{2}$ & $x_{1} x_{2}-x_{3} x_{4}$ \\
\hline $\mathfrak{S}_{8}$ & $T_{4}$ & $x_{7} x_{8}+x_{3} x_{6}+x_{2} x_{5}+x_{1} x_{4}+2\left(x_{6} x_{8}+x_{3} x_{5}+x_{2} x_{4}+x_{1} x_{7}\right)$ \\
\hline $\mathfrak{S}_{8}$ & $T_{47}$ & $x_{1} x_{2} x_{3} x_{4}+x_{5} x_{6} x_{7} x_{8}$ \\
\hline $\mathfrak{S}_{9}$ & $T_{14}$ & $\begin{array}{l}\left(x_{4} x_{6} x_{7} x_{9}+x_{4} x_{5} x_{6} x_{8}+x_{3} x_{5} x_{8} x_{9}+x_{3} x_{5} x_{6} x_{7}+x_{3} x_{4} x_{7} x_{8}+x_{2} x_{7} x_{8} x_{9}+x_{2} x_{5} x_{6} x_{9}+\right. \\
x_{2} x_{4} x_{5} x_{7}+x_{2} x_{3} x_{6} x_{8}+x_{2} x_{3} x_{4} x_{9}+x_{1} x_{6} x_{8} x_{9}+x_{1} x_{5} x_{7} x_{8}+x_{1} x_{4} x_{5} x_{9}+x_{1} x_{3} x_{7} x_{9}+ \\
\left.x_{1} x_{3} x_{4} x_{6}+x_{1} x_{2} x_{6} x_{7}+x_{1} x_{2} x_{4} x_{8}+x_{1} x_{2} x_{3} x_{5}\right)+2\left(x_{4} x_{5} x_{7} x_{8}+x_{4} x_{5} x_{6} x_{9}+x_{3} x_{7} x_{8} x_{9}+\right. \\
x_{3} x_{5} x_{6} x_{8}+x_{3} x_{4} x_{6} x_{7}+x_{2} x_{6} x_{8} x_{9}+x_{2} x_{5} x_{6} x_{7}+x_{2} x_{4} x_{7} x_{9}+x_{2} x_{3} x_{5} x_{9}+x_{2} x_{3} x_{4} x_{8}+ \\
x_{1} x_{6} x_{7} x_{9}+x_{1} x_{5} x_{8} x_{9}+x_{1} x_{4} x_{6} x_{8}+x_{1} x_{3} x_{5} x_{7}+x_{1} x_{3} x_{4} x_{9}+x_{1} x_{2} x_{7} x_{8}+x_{1} x_{2} x_{4} x_{5}+ \\
\left.x_{1} x_{2} x_{3} x_{6}\right)\end{array}$ \\
\hline $\mathfrak{S}_{9}$ & $T_{10}$ & $\begin{array}{l}x_{6} x_{7} x_{8} x_{9}+x_{5} x_{6} x_{7} x_{8}+x_{4} x_{5} x_{8} x_{9}+x_{4} x_{5} x_{6} x_{7}+x_{3} x_{5} x_{7} x_{9}+x_{3} x_{4} x_{8} x_{9}+x_{3} x_{4} x_{7} x_{8}+ \\
x_{3} x_{4} x_{5} x_{6}+x_{2} x_{5} x_{7} x_{9}+x_{2} x_{4} x_{7} x_{9}+x_{2} x_{4} x_{6} x_{9}+x_{2} x_{4} x_{6} x_{8}+x_{2} x_{3} x_{7} x_{8}+x_{2} x_{3} x_{6} x_{7}+ \\
x_{2} x_{3} x_{4} x_{5}+x_{1} x_{7} x_{8} x_{9}+x_{1} x_{5} x_{6} x_{9}+x_{1} x_{4} x_{6} x_{8}+x_{1} x_{4} x_{5} x_{9}+x_{1} x_{3} x_{6} x_{8}+x_{1} x_{3} x_{5} x_{8}+ \\
x_{1} x_{3} x_{5} x_{7}+x_{1} x_{2} x_{8} x_{9}+x_{1} x_{2} x_{6} x_{7}+x_{1} x_{2} x_{5} x_{6}+x_{1} x_{2} x_{3} x_{9}+x_{1} x_{2} x_{3} x_{4}\end{array}$ \\
\hline $\mathfrak{S}_{r}$ & $A_{r} \times \mathfrak{S}_{r}$ & $\prod_{1 \leq i<j \leq r}\left(x_{i}-x_{j}\right)$ \\
\hline
\end{tabular}

TABlEAU 1. Polynômes invariants utilisés dans les calculs des résolvantes et multi-résolvantes. Pour un degré donné $n$, la notation $T_{i}$ désigne les sous-groupes transitifs de $\mathfrak{S}_{n}$ décrits dans [Butler et McKay 1983]. 
Le tableau 1 donne les polynômes invariants que nous avons utilisés dans les calculs des résolvantes et multi-résolvantes. Les invariants ont été calculés par le programme Primitive Invariants [Abdeljaouad 1999].

Calcul de résolvante absolue: algorithmes généraux. Pour $f=x^{6}-243 x^{2}+729$, les temps sont:

\begin{tabular}{|llll|}
\hline Groupe & Algo. 6.10 & Algo. 6.3 & SYM \\
\hline$T_{1}$ & $41^{\prime}$ & $\star$ & $\star$ \\
$T_{2}$ & $41^{\prime}$ & $\star$ & $\star$ \\
$T_{3}$ & $57^{\prime}$ & $\star$ & $\star$ \\
$T_{4}$ & $1 \mathrm{~h} 20^{\prime}$ & $\star$ & $\star$ \\
$T_{5}$ & $1 \mathrm{~h} 30^{\prime}$ & $6 \mathrm{~h}$ & $\star$ \\
$T_{6}$ & $2 \mathrm{~h}$ & $\star$ & $\star$ \\
$T_{12}$ & $2 \mathrm{~h}$ & $5 \mathrm{~h} 55^{\prime}$ & $7 \mathrm{~h} 7^{\prime}$ \\
$T_{14}$ & $11^{\prime}$ & $6 \mathrm{~h}$ & $\star$ \\
$\mathfrak{S}_{2} \times \mathfrak{S}_{2} \times \mathfrak{S}_{2}$ & $3^{\prime} 10^{\prime \prime}$ & $3^{\prime} 30^{\prime \prime}$ & $6^{\prime} 8^{\prime \prime}$ \\
\hline
\end{tabular}

$\star$ : calcul non terminé.

Calcul de résolvante absolue: algorithmes spécialisés. On prend maintenant le cas des invariants de faible arité. Fixons $f=x^{7}+8 x^{6}-5 x^{4}-18 x^{2}+x-1$. Les temps sont :

\begin{tabular}{|llll|}
\hline Groupe & Algo. 6.10 & Algo. 6.3 & SYM \\
\hline$x_{1} x_{2}-x_{3} x_{4}$ & $3^{\prime} 10^{\prime \prime}$ & & $2,5^{\prime \prime}$ \\
$x_{1}+2 x_{2}+3 x_{3}$ & $171^{\prime \prime}$ & $128^{\prime \prime}$ & $2 \mathrm{~h} 23^{\prime}$ \\
$x_{1} x_{2}^{2} x_{3}^{3}$ & $18^{\prime} 26^{\prime \prime}$ & $48^{\prime}$ & $16^{\prime} 16^{\prime \prime}$ \\
$x_{1} x_{2} x_{3}$ & $200^{\prime \prime}$ & $250^{\prime \prime}$ & $2,5^{\prime \prime}$ \\
\hline
\end{tabular}

Calculs de multi-résolvantes absolues. Fixons

$$
F=\left(f^{(1)}, f^{(2)}, f^{(3)}, f^{(4)}\right)
$$

où :

$$
\begin{aligned}
f^{(1)}=x^{3} & +6095519139889209290 x^{2} \\
& +14888192843637255648 x \\
& +3495901233607471790 \\
f^{(2)}=x^{3} & +17595319154460550344 x^{2} \\
& +11186788950436463054 x \\
& +11049608948604938510 \\
f^{(3)}=x+ & 8358981594339387494 \\
f^{(4)}=x & +4843668258729955558
\end{aligned}
$$

Les temps mesurés sont les suivants:

\begin{tabular}{|lll|}
\hline Groupes & Algorithme 7.2 & SYM \\
\hline$A_{4} \times \mathfrak{S}_{4}$ & $0,27^{\prime \prime}$ & $15 \mathrm{~h} 20^{\prime}$ \\
$T_{47}$ & $0,06^{\prime \prime}$ & $\star$ \\
$T_{4}$ & $5,46^{\prime \prime}$ & $\star$ \\
\hline
\end{tabular}

$\star$ : temps de calcul supérieur à 2 jours.

Fixons maintenant $P=\left(p^{(1)}, p^{(2)}, p^{(3)}, p^{(4)}\right)$ où :

$$
\begin{aligned}
p^{(1)}=x^{4} & +119 x^{3}+442 x^{2}+x-1 \\
p^{(2)}=x^{3} & +17595319154460550344 x^{2} \\
& +11186788950436463054 x \\
& +11049608948604938510 \\
p^{(3)}=x & +8358981594339387494 \\
p^{(4)}=x & +4843668258729955558
\end{aligned}
$$

\begin{tabular}{|ll|}
\hline Groupes & Algorithme 7.2 \\
\hline$T_{14}$ & $0,05^{\prime \prime}$ \\
$T_{10}$ & $1 \mathrm{~h} 28^{\prime}$ \\
\hline
\end{tabular}

\section{CONCLUSIONS}

Cet article a montré comment éviter les deux principaux défauts des méthodes de calcul de résolvantes basées sur le résultant (facteurs parasites et puissances superflues). L'algorithme auquel nous avons abouti (algorithme 6.10) a permis de calculer des résolvantes qui n'étaient pas calculables par d'autres méthodes (par exemple: la résolvante de $T_{1}$ en degré 6 ). Les algorithmes spécialisés implantés dans SYM peuvent être beaucoup plus rapides (cas des résolvantes produits, symétriques) mais ce n'est pas toujours le cas (résolvantes linéaires, résolvantes monomiales). Pour les invariants de grande arité, les temps de calcul restent élevés et au delà du degré 7, de nombreux exemples n'aboutissent pas. En revanche, l'algorithme de multi-résolvante (algorithme 7.2 ) est très efficace.

\section{DISPONIBILITÉ ELECTRONIQUE}

Les rapports internes et techniques sont disponibles sur le serveur http://www.lip6.fr/reports/ . 


\section{BIBLIOGRAPHIE}

[Abdeljaouad 1999] I. Abdeljaouad, "Calculs d'invariants primitifs de groupes finis", Theor. Inform. Appl. 33:1 (1999), 59-77.

[Ampère 1826] A.-M. Ampère, Fonctions interpolaires, Annales de M.Gergonne, 1826.

[Arnaudiès 1989] J.-M. Arnaudiès, Elimination, Ph.D. thesis, Universit Paul Sabatier de Toulouse, 1989. à paraître dans Adv. in Math.

[Arnaudiès 1992] J.-M. Arnaudiès, "Théorème de Bézout, formule de Poisson-Perron et courbes rationnelles planes génériques", Technical Report 92-24, LITP, 1992.

[Arnaudiès et Valibouze 1997] J.-M. Arnaudiès et A. Valibouze, "Lagrange resolvents", J. Pure Appl. Algebra 117/118 (1997), 23-40.

[Becker et Weispfenning 1993] T. Becker et V. Weispfenning, Gröbner bases: A computational approach to commutative algebra, Springer, New York, 1993. In cooperation with Heinz Kredel.

[Butler et McKay 1983] G. Butler et J. McKay, "The transitive groups of degree up to eleven", Comm. Algebra 11:8 (1983), 863-911.

[Cauchy 1882] A. L. Cauchy, "Usage des fonctions interpolaires dans la détermination des fonctions symétriques des racines d'une équation algébrique donnée", pp. 473 Gauthier-Villars, Paris, 1882.

[Eichenlaub et Olivier 1996] Y. Eichenlaub et M. Olivier, "GALP: un programme pour calculer des groupes de Galois", Technical report, 1996. Voir http://www.math.u-bordeaux.fr/A2X/Logiciels.html.

[Girstmair 1983] K. Girstmair, "On the computation of resolvents and Galois groups", Manuscripta Math. 43:2-3 (1983), 289-307.

[Henrici 1956] P. Henrici, "Automatic computations with power series", J. Assoc. Comput. Mach. 3 (1956), 1015.
[Jenks et Sutor 1992] R. D. Jenks et R. S. Sutor, AXIOM, The scientific computation system, Numerical Algorithms Group Ltd., Oxford, 1992.

[Lagrange 1770] J.-L. de Lagrange, "Réflexions sur la résolution algébrique des équations", (1770). Reprinted as pp. 205-421, v. 4, in Euvres de Lagrange, edited by J.-A. Serret, Paris, Gauthier-Villars.

[Lascoux et Pragacz 1988] A. Lascoux et P. Pragacz, " $S$ function series", J. Phys. A Math. Gen. 21:22 (1988), 4105-4114.

[Lehobey 1997] F. Lehobey, "Resolvent computations by resultants without extraneous powers", dans ISSAC 9\%: Proceedings, édité par W. Küchlin, ACM Press, New York, 1997.

[Maxima $\geq 1999$ ] Maxima, "MAXIMA", Voir http:// www.ma.utexas.edu/maxima.html. Common Lisp implementation of Macsyma, maintained by William Schelter.

[Schönert et al. 1995] M. Schönert et al., GAP: Groups, algorithms, and programming, $5 \mathrm{e}$ éd., Lehrstuhl D für Mathematik, RWTH Aachen, 1995. Voir http:// www-gap.dcs.st-and.ac.uk/ gap.

[Soicher 1984] L. H. Soicher, "An algorithm for computing Galois groups", pp. 291-296 dans Computational group theory (Durham, 1982), édité par M. D. Atkinson, Academic Press, London, 1984.

[Stauduhar 1973] R. P. Stauduhar, "The determination of Galois groups", Math. Comp. 27 (1973), 981-996.

[Valibouze 1989] A. Valibouze, "SYM, Symbolic computation with symmetric polynomials: an extension to MACSYMA", pp. 308-320 dans Computers and mathematics (Cambridge, MA, 1989), édité par Kaltofen, Erich and Watt, Stephen M., Springer, New York, 1989.

[Valibouze 1999] A. Valibouze, "Etude des relations algébriques entre les racines d'un polynôme d'une variable", Bull. Belgian Math. Soc. 6:4 (1999), 507535 .

Nicolas Rennert, LIP6, case 167, Université Paris VI, 4 place Jussieu, 75252 Paris Cedex 05, France (Nicolas.Rennert@lip6.fr)

Annick Valibouze, LIP6, case 167, Université Paris VI, 4 place Jussieu, 75252 Paris Cedex 05, France (avb@medicis.polytechnique.fr, Annick.Valibouze@lip6.fr) 